\title{
Dynamics of Water Molecules and Ions in Concentrated Lithium Chloride Solutions Probed with Ultrafast 2D IR Spectroscopy
}

\author{
Rongfeng Yuan, and Michael Fayer* \\ Department of Chemistry \\ Stanford University, Stanford, CA 94305 \\ *phone: 650 723-4446; email: fayer@stanford.edu
}

\section{Supporting Information}

\section{Discussion of Band Shape Analysis Method}

\subsection{Situations where applying the new method is necessary.}

In the main text, we described a method that enabled us to extract the correct CLS from 2D spectra where peaks are severely overlapped. Here we want to briefly discuss when the method is needed. In the following discussion, we generated 2D spectra by calculating response function with a set of FFCF parameters, vibrational lifetimes, chemical exchange parameters, transition dipoles, etc. ${ }^{1,2}$ Then we applied the CLS method on the simulated 2D spectra without considering the overlapping peaks and compared the result with the correct CLS, which can be obtained through simulating only a single component. To make our discussion concise, we chose to set the two components in calculation to share all FFCF parameters (see Table S1), lifetimes and transition dipoles etc., except when noted. A real situation can be far more complicated. For the two components under focus, the FFCF parameters and vibrational lifetime do not have to be the same. They may have different anharmonicities. But the purpose of this discussion is not to quantify the extent of deviation but to provide general criteria on which one can decide whether to consider the effect the overlapping peaks. We think the current comparison serves this purpose well enough.

Table S1. FFCF parameters used in 2D Spectra generation

\begin{tabular}{|c|c|c|c|c|}
\hline$\Gamma\left(\mathrm{cm}^{-1}\right)$ & $\Delta_{1}\left(\mathrm{~cm}^{-1}\right)$ & $\tau_{1}(\mathrm{ps})$ & $\Delta_{2}\left(\mathrm{~cm}^{-1}\right)$ & $\tau_{2}(\mathrm{ps})$ \\
\hline 4.7 & 5.3 & 2.3 & 2.5 & 28 \\
\hline
\end{tabular}


The first and most obvious factor is the spectral separation $\Delta$ of the two components under study relative to their full-width-at-half-maximums ( $\Delta$ FWHM). If $\Delta>>\Delta$ FwHM, there should be no interference in extracting the correct CLS. If $\Delta<\Delta$ FWHM, a deviation from real value is likely to happen if the CLS method is directly applied. To avoid complication of other factors, we do not include chemical exchange in the calculation and set the anharmonicity between 0-1 and 1-2 transitions to be very large $\left(100 \mathrm{~cm}^{-1}\right)$. Fig. S1A shows the change of extracted CLS as $\Delta$ decreases relatively to a fixed $\Delta_{\text {FWHM. }}$ It is obvious that the distortion is negligible until $\Delta=1.14 \Delta_{\text {FWHM, }}$ in which case there is very small yet noticeable difference between the extracted CLS and the correct CLS. All of the CLS are fit with biexponential decays and the fitting parameters are summarized in Table S2. Considering the common experimental noise level, a reasonable boundary can be set at $\Delta=1.1 \Delta$ FWHM. This general condition can be used to determine if additional consideration needs to be made when using CLS method.
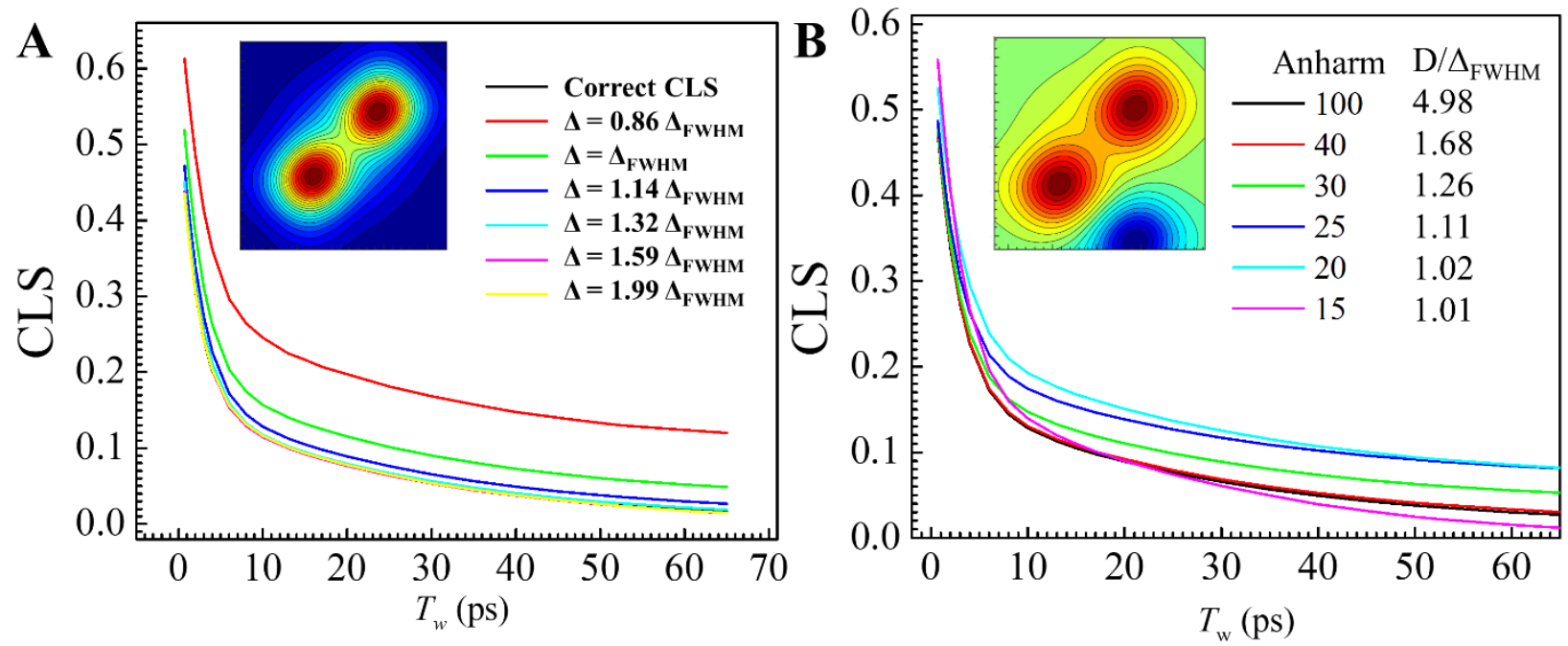

Figure S1. Numerical Calculation shows the CLS deviation due to the effect of (A) overlapping diagonal peaks and (B) overlapping between a lower-frequency 0-1 transition and the 1-2 transition of a higher-frequency mode. (A) The black curve represent the correct CLS, which is hardly visible due to its overlapping to conditions from $\Delta=1.32-1.99 \Delta_{\text {FWHм}}$. The deep blue curve shows a slight difference while the green curve presents significant difference. The inset 2D spectrum shows the $\Delta=\Delta_{\text {FWHM }}$ at $T_{w}=10 \mathrm{ps}$. (B)The black curve corresponds to the largest $\mathrm{D} / \Delta_{\text {FWHM }}$ ratio, meaning smallest $1-2$ influence. As the ratio decreases, the CLS deviation starts to show up and increases. The inset $2 \mathrm{D}$ spectrum shows the $\Delta=1.14 \Delta_{\mathrm{FWHM}}$ and $\mathrm{D} / \Delta_{\mathrm{FWHM}}=1.26$ at $T_{w}=10 \mathrm{ps}$. 
Table S2. Fitting parameters of biexponential decay shown in Fig. S1A.

\begin{tabular}{lrrrr}
\hline & \multicolumn{1}{c}{$\mathrm{A}_{1}{ }^{\mathrm{b}}$} & $\tau_{1}(\mathrm{ps})$ & $\mathrm{A}_{2}$ & $\tau_{2}(\mathrm{ps})$ \\
\hline Correct $^{\mathrm{a}}$ & $0.386 \pm 0.003^{\mathrm{c}}$ & $2.26 \pm 0.04$ & $0.156 \pm 0.002$ & $28.4 \pm 0.6$ \\
$\Delta=0.86 \Delta_{\text {FWHM }}$ & $0.458 \pm 0.002$ & $3.03 \pm 0.04$ & $0.253 \pm 0.002$ & $78.7 \pm 1.3$ \\
$\Delta=\Delta_{\text {FWHM }}$ & $0.447 \pm 0.003$ & $2.65 \pm 0.04$ & $0.179 \pm 0.002$ & $45.9 \pm 0.8$ \\
$\Delta=1.14 \Delta_{\text {FWHM }}$ & $0.417 \pm 0.003$ & $2.51 \pm 0.04$ & $0.159 \pm 0.002$ & $34.6 \pm 0.7$ \\
$\Delta=1.32 \Delta_{\text {FWHM }}$ & $0.401 \pm 0.003$ & $2.41 \pm 0.04$ & $0.153 \pm 0.002$ & $30.2 \pm 0.6$ \\
$\Delta=1.59 \Delta_{\text {FWHM }}$ & $0.389 \pm 0.003$ & $2.27 \pm 0.04$ & $0.157 \pm 0.002$ & $27.7 \pm 0.6$ \\
$\Delta=1.99 \Delta_{\text {FWHM }}$ & $0.382 \pm 0.003$ & $2.24 \pm 0.04$ & $0.160 \pm 0.002$ & $27.5 \pm 0.6$ \\
\hline
\end{tabular}

a. Correct refers to the condition with very large spectral separation and has not aberration to the CLS.

b. $A_{i}$ and $t_{i}$ are amplitude and time constant respectively in the biexponential fit, where $i=1,2$.

c. The errors for all fitting parameters are fitting errors. Naturally, it is very small because the 2D spectra are generated by numerical calculation.

The second factor is anharmonicity between the $0-1$ and 1-2 transition. It was previously shown that for a single component, the existence of 1-2 transition does not affect the extracted CLS. ${ }^{3}$ This, however, does not prevent the 1-2 transition of a higher-frequency component from affecting the 0-1 transition band shape of a lower-frequency component, if the spectral separation and anharmonicity are in a good match. This is exactly the case in the current sample. Here, the spectral separation is $\sim 17 \mathrm{~cm}^{-1}$ and the anharmonicity is $\sim 25 \mathrm{~cm}^{-1}$. As one can see from the Fig. 2 in the main text, the overlap between the L component's 1-2 and W component's $0-1$ is severe. Now, because the effect of anharmonicity is also highly dependent the spectral separation of the two 0-1 transition, we defined a new variable, $\mathrm{D}$, which stands for the distance between the peak positions on a $2 \mathrm{D}$ spectrum. This means, for example, if the peak positions of two species differs by $15 \mathrm{~cm}^{-1}$ on a linear FT-IR spectrum, D on a 2D spectrum is $15 \times \sqrt{2}=21.2 \mathrm{~cm}^{-1}$. In an example shown in Fig. S1 B, we use our experimental number between $\mathrm{W}$ and $\mathrm{L}$ states for spectral separation $\Delta=17 \mathrm{~cm}^{-1}$. We used the same FFCF parameters as in Table S1. The difference is that we used a series of anharmonicities and this creates a series of $\mathrm{D}$ between the 1-2 transition of $\mathrm{L}$ component and 0-1 transition of $\mathrm{W}$ component, shown in Fig. S1B. The anharmonicities and the ratios between $\mathrm{D}$ and $\triangle$ FWHM are shown on the plot as well. CLS was extracted on the bottom left 
W peaks (diagonal peaks with lower frequency). The CLSs were fit with biexponential decays and the fitting parameters are summarized in Table S3. It is evident that when $\mathrm{D}<1.4 \Delta$ FwHM, the effect is generally non-negligible. Depending on the position of L 1-2 transition, whether on the upperright or bottom right of the $\mathrm{W}$ 0-1 transition, the aberrations are both significant but distinct.

Table S3. Fitting parameters of biexponential decay shown in Fig. S1B

\begin{tabular}{ccccc}
\hline $\mathrm{D} / \Delta_{\text {FWHM }}$ & $\mathrm{A}_{1} \mathrm{~b}$ & $\tau_{1}(\mathrm{ps})$ & $\mathrm{A}_{2}$ & $\tau_{2}(\mathrm{ps})$ \\
\hline $4.98^{\mathrm{a}}$ & $0.417 \pm 0.004^{\mathrm{c}}$ & $2.50 \pm 0.05$ & $0.159 \pm 0.003$ & $34.6 \pm 0.9$ \\
1.68 & $0.418 \pm 0.004$ & $2.53 \pm 0.05$ & $0.159 \pm 0.003$ & $36.7 \pm 0.9$ \\
1.26 & $0.414 \pm 0.004$ & $2.55 \pm 0.05$ & $0.164 \pm 0.003$ & $51.6 \pm 1.3$ \\
1.11 & $0.392 \pm 0.004$ & $2.67 \pm 0.05$ & $0.185 \pm 0.003$ & $70.7 \pm 1.8$ \\
1.02 & $0.408 \pm 0.004$ & $2.87 \pm 0.05$ & $0.206 \pm 0.003$ & $63.9 \pm 1.5$ \\
1.01 & $0.491 \pm 0.004$ & $2.54 \pm 0.05$ & $0.194 \pm 0.003$ & $25.1 \pm 0.6$
\end{tabular}

a. The ratio roughly determines the effect of 1-2 transition on a lower-frequency $0-1$ mode. When the ratio is 5 , there is basically not effect of 1-2 transition.

b. $A_{i}$ and $t_{i}$ are amplitude and time constant respectively in the biexponential fit, where $i=1,2$.

c. The errors for all fitting parameters are fitting errors. Naturally, it is very small because the 2D spectra are generated by numerical calculation.

The third factor is chemical exchange. This effect is a combination of the first two factors discussed except that the interfering peaks' $T_{\mathrm{w}}$-dependent relative volumes. Real situations can be very diverse due to the vast variety of chemical exchange timescale, spectra separation and the size of anharmonicities.

To conclude this section, we have discussed three potential interfering scenarios and provide a general qualitative guidance on the potential aberrations. Real experimental situations can be very diverse and our discussion is obviously not all-encompassing. One factor that we did not elaborate on is the relative intensity of the two peaks. This factor will change the amount of deviation from the real CLS.

\subsection{Validation of the 2D Gaussian Subtraction method}

In this section, we generated ideal $2 \mathrm{D}$ spectra by response function calculation. ${ }^{1-3}$ We can therefore arbitrarily change the peak separation, FFCF parameters or chemical exchange 
parameters, and use our proposed method to test whether we can reproduce the original FFCF settings.

\subsubsection{Two closely spaced peaks without chemical exchange}

In this section, we used the same FFCF parameters for both peaks, shown in Table S1. The anharmonicities are set to be $100 \mathrm{~cm}^{-1}$ and there is no chemical exchange. Due to the resullts shown in Fig. S1A, we decided to use two cases that would have large CLS deviation if this method is not applied: $\Delta=\Delta_{\mathrm{FWHM}}$ and $\Delta=0.86 \Delta_{\mathrm{FWHM}}$, which correspond to $\mathrm{A}$ and $\mathrm{B}$ in Fig. S2. In both of the figures, black lines are the original intended CLS. The red lines are the extracted CLS if it is directly extracted from the 2D spectra. The blue lines are the extracted CLS using the method described in the main text. Though the efficacy becomes worse as the spectral separation becomes smaller, it is obvious that our method greatly improves the reproduction of the intended CLS. Even in scenario B, our method could reduce the error from $290 \%$ to $40 \%$. The remaining error probably originates from the fact that Gaussian functions cannot fully reproduce the real line shape, especially the Lorentzian homogeneous component.
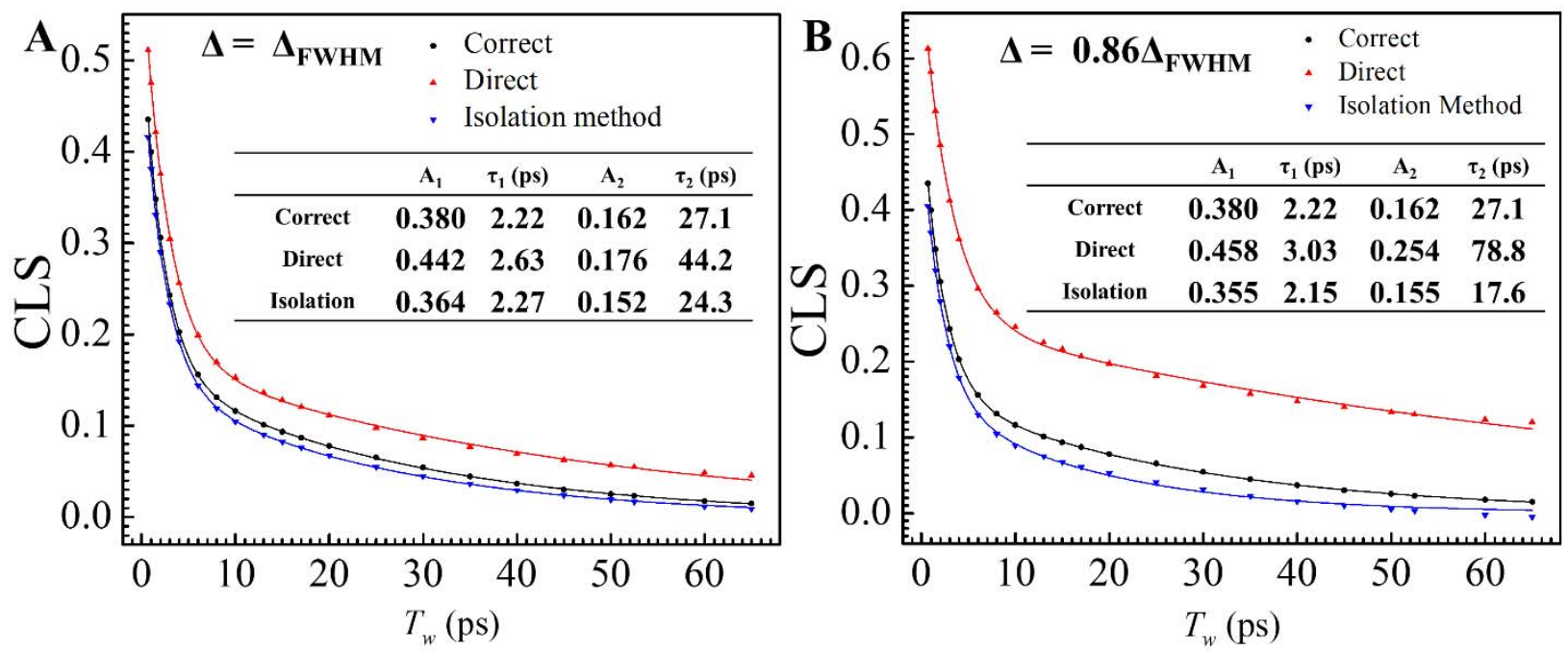

Figure S2. Use numerical calculation method to test the method with known results. Here we show situations with $\Delta=\Delta_{\mathrm{FWHM}}$ and $\Delta=0.86 \Delta_{\mathrm{FWHM}}$, corresponding to A and B, respectively. In both of the figures, black, red and blue refers to the correct CLS, the CLS directly obtained from complete 2D spectra and the CLS extracted using our proposed method. The dots are CLS obtained at a series of $\mathrm{T}_{\mathrm{w}} \mathrm{s}$ and the lines are biexponential fit. The fitting results are included in the inset tables. In the tables, "correct", "direct" and "isolation" represents CLS that is correct without aberration, CLS that was extracted directly on the overlapping 2D spectra, and CLS that was extracted using our 2D subtration method, respectively. 


\subsubsection{Effect of homogeneous component}

In this section, we would like to see whether the amount of the homogeneous component will affect the efficacy of our procedure because a Gaussian function cannot perfectly reproduce a Voigt line shape with a Lorentzian component. Therefore, we generated two other sets of 2D spectra for the case $\Delta=0.86 \Delta$ FWHM. In these sets, the homogeneous component is greatly increased or decreased while the inhomogeneous components' amplitudes are adjusted accordingly to maintain the same FWHM, the parameters of which is shown in Table S4.

Table S4. Settings of FFCF parameters for examining the effect of homogeneous component

\begin{tabular}{cccccc}
\hline Name & $\Gamma\left(\mathrm{cm}^{-1}\right)$ & $\Delta_{1}\left(\mathrm{~cm}^{-1}\right)$ & $\tau_{1}(\mathrm{ps})$ & $\Delta_{2}\left(\mathrm{~cm}^{-1}\right)$ & $\tau_{2}(\mathrm{ps})$ \\
\hline NoHomo & 0 & 6.4 & 2.3 & 3 & 28 \\
Middle & 4.7 & 5.3 & 2.3 & 2.5 & 28 \\
MoreHomo & 8.4 & 4.2 & 2.3 & 2 & 28 \\
\hline
\end{tabular}
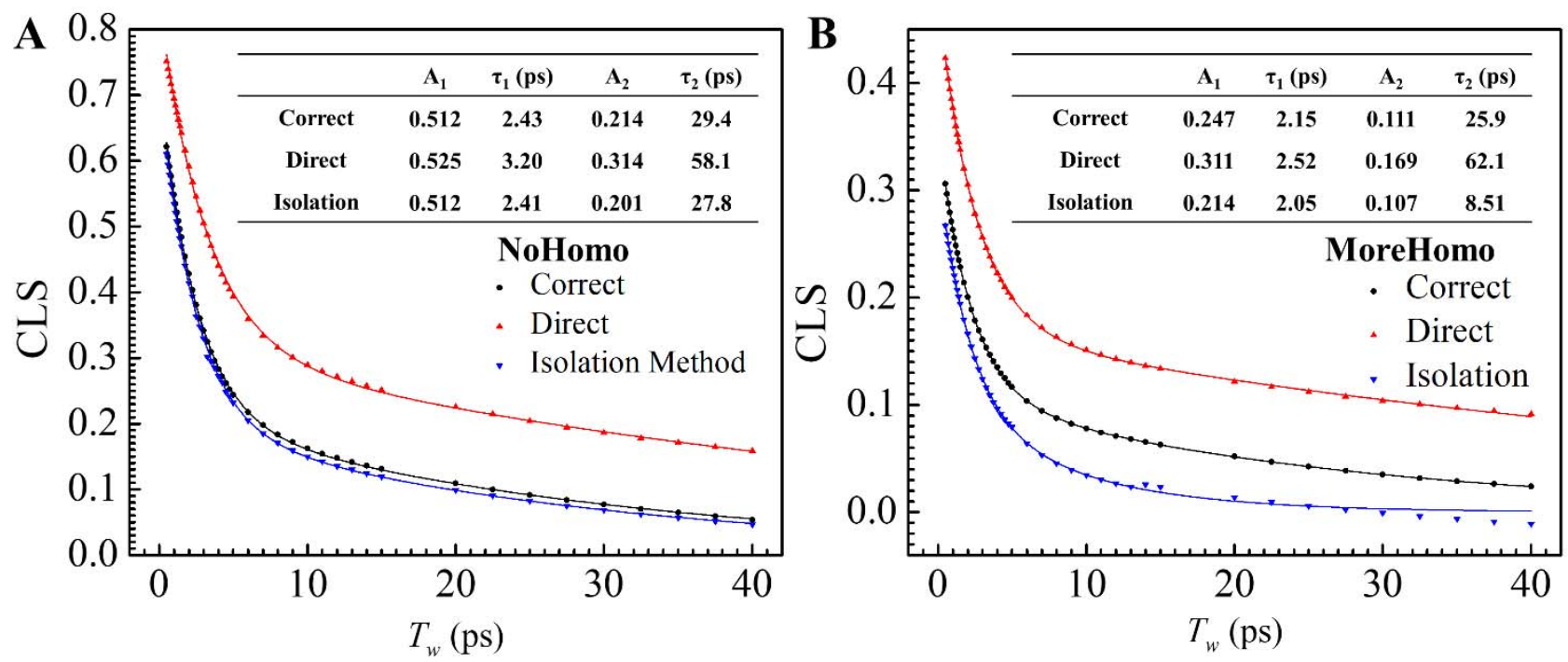

Figure S3. Test the effect of homogeneous component on the method. In both A and B, dots are CLS obtained at a series of $T_{\mathrm{w}} \mathrm{s}$ and the lines are biexponential decay fitting results. The fitted parameters are presented in the inset table where $A_{i}$ and $\tau_{i}(i=1,2)$ stands for the amplitude and time constant, respectively. A corresponds to the "NoHomo" condition, of which the FFCF parameters are specified in Table S4. Clearly, our method can reproduce the correct CLS extremely well. B corresponds to the "MoreHomo" condition. Our method yields a less satisfactory result due to the mismatch of Gaussian and the real line shape with a high Lorentzian component.

As shown in Fig. S3, combined with B in Fig. S2, it is clear that the homogeneous component plays an important factor. As the homogeneous component gets smaller, 2D Gaussian 
functions can reproduce the spectra better, leading to more accurate reproduction of the intended CLS. A Gaussian function cannot generate the slowly decreasing tail of a Lorentzian line shape and the algorithm tries to compensate for it by increasing the width of Gaussian function, thus causing a deviation from the real shape. For a perfect 2D spectrum with no noise, one may use a Voigt function to better reproduce the experimental line shape. However, for most experimental data, a Voigt function is not practical because it has two linewidth parameters that can compensate for each other and its Lorentzian component is especially susceptible to regions where the signalto-noise ratio is poor because a Lorentzian has a much slower decaying tail region than a Gaussian function.

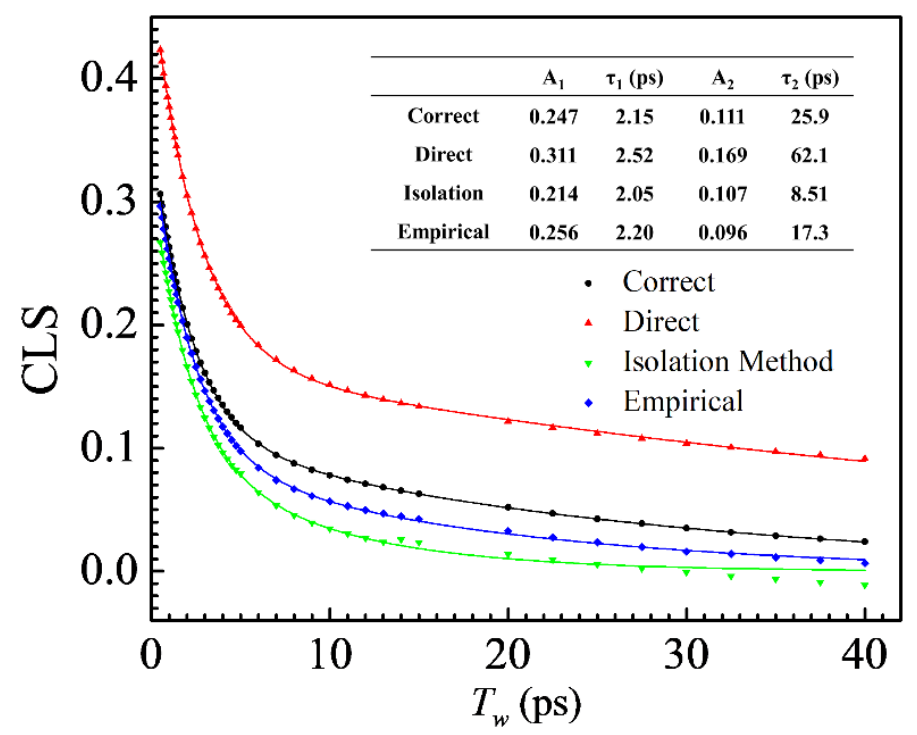

Figure S4. Effect of the empirical method that adds cross peaks to simulate Lorentzian components. The black curve is the correct or intended CLS. The red curve is obtained directly on the 2D spectra without isolating the peak. Green and blue curves are obtained through the standard and empirical method, respectively. Except for the blue curve, other lines are the same as Fig. S3 B. The inset table contains the fitting parameters of biexponential decay. Obviously, the empirical method, which adds cross peak to the fitting routine, greatly improves the effectiveness of this method.

In an experimental 2D spectra, the homogeneous (Lorentzian) component usually leads to a star-shaped pattern and this leads to a cross-peak-like pattern if the two components are close enough diagonally. Empirically, we found that one can improve the reproduction of the intended CLS by fitting 2D spectra with cross peaks that can simulate the extended Lorentzian components. This partially prevents the algorithm from increasing the Gaussian width to simulate the Lorentzian tail, which causes fitting deviation mentioned above. The widths of the cross peak can be fixed to the experimental IR absorption linewidth and only the amplitude is a variable. Shown in Fig. S4, 
this empirical method is applied to situation with $\Delta=0.86 \Delta$ FwHM and the set FFCF parameters with the most homogeneous component. The blue curve (empirical method) can reproduce the CLS better than the green curve (standard method without cross peaks).

\subsubsection{Test the simulation result with chemical exchange and 1-2 transition}

The calculation tests for the factors of chemical exchange and 1-2 transition have too many degrees of freedom and are difficult to summarize. Thus, we decided to test using parameters similar to the experimental condition. We used the FFCF and chemical exchange parameters that are similar to the dynamics of $\mathrm{W}$ and $\mathrm{L}$ components in $\mathrm{LiCl} 1: 4 \mathrm{H}_{2} \mathrm{O}$ solution to generate 2D spectra. Then we used our proposed method to extract the FFCF for structural dynamics and compare it with our initial parameters. The comparison is shown in Fig.S5.
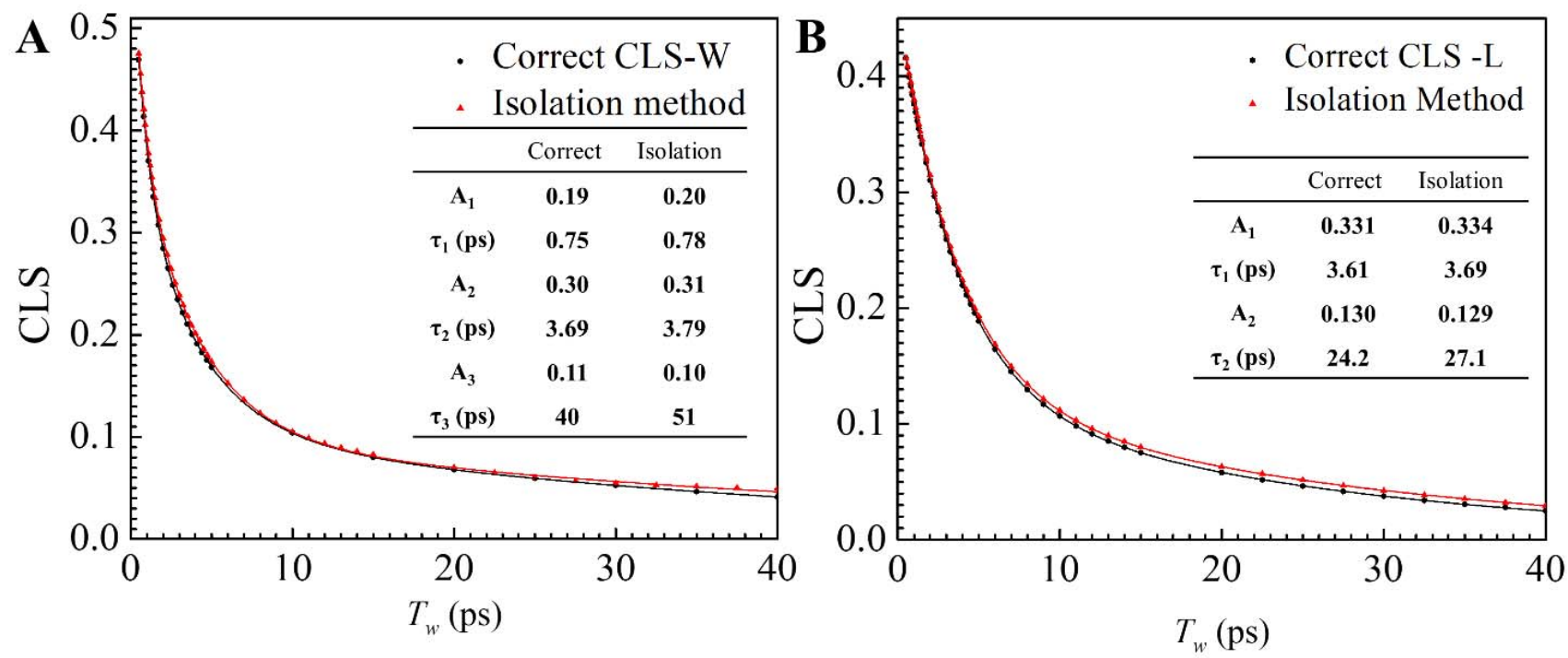

Figure S5. We tested the isolation method on a system that is similar to our experimental system. A and B corresponds to $\mathrm{W}$ and $\mathrm{L}$ components, respectively. In each figure, black curve corresponds the correct or intended CLS and the red curve shows the extracted CLS by our method. Clearly, this method works extremely well on our system. Exponential fitting results are presented in the inset tables.

Evidently, our proposed method can reproduce the intended CLS extremely well. A and B corresponds to $\mathrm{W}$ and $\mathrm{L}$ components, respectively. In both figures, the red line (CLS extracted using our proposed method) is very close to the black line (the intended or setting CLS). This result demonstrates that our proposed method can extract the structural dynamics well.

\subsection{CLS distortion caused by overlapping peaks}

In Fig. S6, we plotted the CLS extracted directly with raw experimental 2D spectra with overlapping peaks (black dots) and the CLS extracted with our method (red dots). Not only is there 
great difference before $25 \mathrm{ps}$, the CLS of latter $T_{w}$ s was severely distorted due to the band shape aberration as well. Therefore, our method brings significant improvement to the extraction of spectral diffusion.

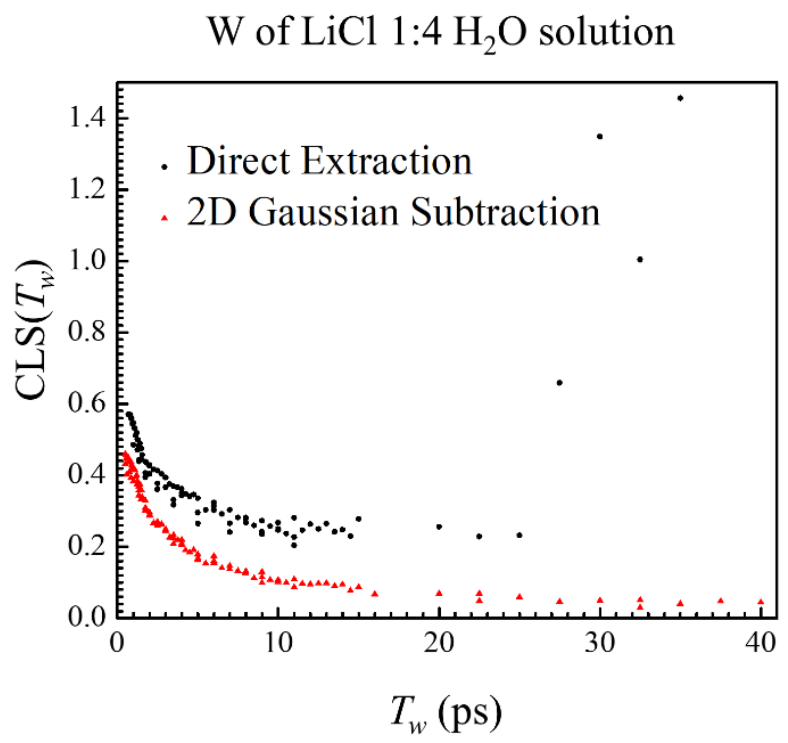

Figure S6. The black and red dots are both experimental data of CLS extracted for W component in LiCl 1:4 $\mathrm{H}_{2} \mathrm{O}$ solution. Direct application of the CLS method without considering the overlapping peaks yields black dots. The red dots are CLS extracted from the isolated $\mathrm{W}$ component using our presented method in the text.

\section{Validation of anisotropy extracting method}

To test our proposed method, we chose to use the system of MeSCN in pure water where there is only one component and PSPP measurement can be conducted directly to extract the anisotropy. The experimental 2D spectra of this system was directly projected onto the $\omega_{m}$ axis. Anisotropy was then calculated based on the method introduced in Section C in main text. In Fig. S7, we plotted the extracted anisotropy from 2D spectra projection method and from PSPP measurements. 


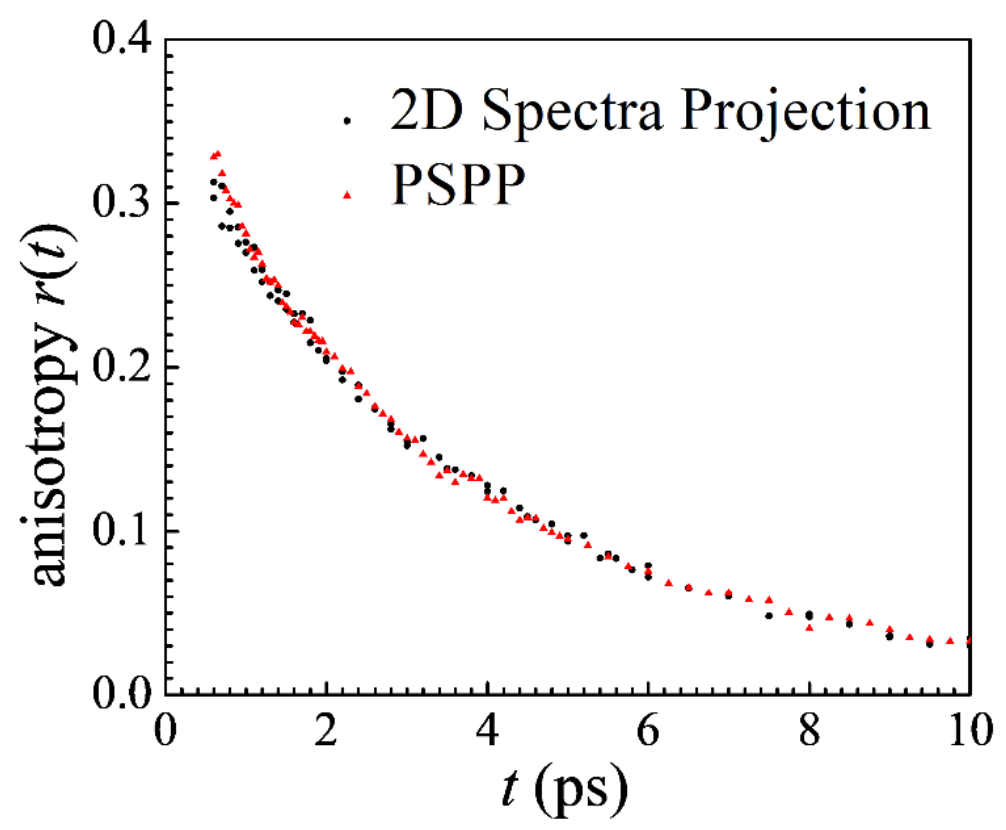

Figure S7. The red dots are the experimental anisotropy extracted from pump-probe (PSPP) measurement. The black dots are extracted from 2D spectra. They are basically on top of each other except for the very early $T_{w}$ region.

It is clear from Fig. S7 that this method could reproduce the anisotropy extremely well overall. The only small deviation happens at the early $T_{w}$ region. This difference, we believe, originates from the $\tau$-scanning process during the 2D IR data acquisition process.

In 2D IR spectroscopy, there are three input pulses, two pump pulses and one probe pulse. The interactions leads to the emission of the fourth vibrational echo pulse. The time delays between the four pulses are $\tau, T_{w}, t_{3}$ in sequence. $t_{3}$ is the time delay between the third pulse and the vibrational echo, which is smaller or equal to $\tau$. The relationship between signal and polarization can be summarized as the following two equations ${ }^{4}$

$$
\begin{aligned}
& S_{\|}=\frac{1}{9} P(t) C_{1}(\tau)\left(1+\frac{4}{5} C_{2}\left(T_{w}\right)\right) C_{1}\left(t_{3}\right) \\
& S_{\perp}=\frac{1}{9} P(t) C_{1}(\tau)\left(1-\frac{2}{5} C_{2}\left(T_{w}\right)\right) C_{1}\left(t_{3}\right)
\end{aligned}
$$

where the $C_{1}$ and $C_{2}$ are the first-order and second-order Legendre polynomial orientational correlation function, respectively. For a sphere with a rotational diffusion coefficient of $D_{\text {or, }}$ $C_{1}(t)=\exp \left(-t / 2 D_{o r}\right)$ and $C_{2}(t)=\exp \left(-t / 6 D_{o r}\right)$. 
For PSPP measurement, $\tau=t_{3}=0$ and the equation above becomes eq. 5 in the main text. Rotational diffusion only happens during the $T_{w}$ period. For 2D IR measurement, however, $\tau$ and $t_{3}$ are not 0 . Generally, the contribution of $C_{1}$ is ignored due to the common short $\tau$ scan and relatively slow reorientation relaxation. For MeSCN in pure water, however, this practice is not a great approximation when $T_{w}$ is small. When measured directly with PSPP, the anisotropy decay is fit with a biexponential decay function and the two time constants are $1.2 \mathrm{ps}$ and $4.7 \mathrm{ps} .^{5}$ This means that for $C_{1}$ function, these time constants become $3.6 \mathrm{ps}$ and $15.1 \mathrm{ps}$. The $\tau$ scan in 2D IR acquisition, on the other hand, requires scanning up to $4 \mathrm{ps}$. The decay of $C_{1}$ at $\tau=4 \mathrm{ps}$ is no longer negligible for the fast wobbling component. However we do want to stress that one 2D spectrum requires the signal of $\tau$ from 0 to $4 \mathrm{ps}$, so it is only the later $\tau$ scan signal that will cause the deviation from pure $C_{2}$ correlation function. In practice, this effect is pretty small given that the two curves in Fig. S7 are basically on top of each other after 1 ps. This effect should be even smaller or negligible for $\mathrm{MeSCN}$ in concentrated $\mathrm{LiCl}$ solution given the much slower reorientation relaxation.

\section{Simulation validation of chemical exchange induced spectral diffusion}

In section B of main text, we introduced a method of removing the chemical-exchangeinduced spectral diffusion from the structural spectral diffusion of the diagonal peaks. In this proposed method, $C L S_{T_{w}}^{S S D}=\mathrm{CLS}_{T_{w}}^{E x p} / x_{T_{w}}^{S S D}$ is used. We want to test whether this equation is valid for $2 \mathrm{D}$ spectra generated from numerical calculation. We applied the same simulation method used in Section 1. In this simulation, we used similar FFCF and chemical exchange parameters measured in the sample of MeSCN in $\mathrm{LiCl} \mathrm{1:4} \mathrm{H}_{2} \mathrm{O}$ solution. Based on the chemical exchange parameters, $x_{T_{w}}^{S S D}$ can be obtained from the exchange kinetic model based on Section B of main text and our previous publication ${ }^{5}$. From the simulated 2D spectra with and without chemical exchange, $C L S_{T_{w}}^{S S D}$ and $\mathrm{CLS}_{T_{w}}^{E x p}$ can be extracted, respectively. Note in this simulation, we set the peak separation to be very large so that the band shape of diagonal peaks are not distorted by overlapping peaks. Figure S8 plots the comparison between $x_{T_{w}}^{S S D}$ and $\operatorname{CLS}_{T_{w}}^{E x p} / C L S_{T_{w}}^{S S D}$ for W and L components. The two values are basically on top of each other, supporting the derivation presented in Section B of main text. 

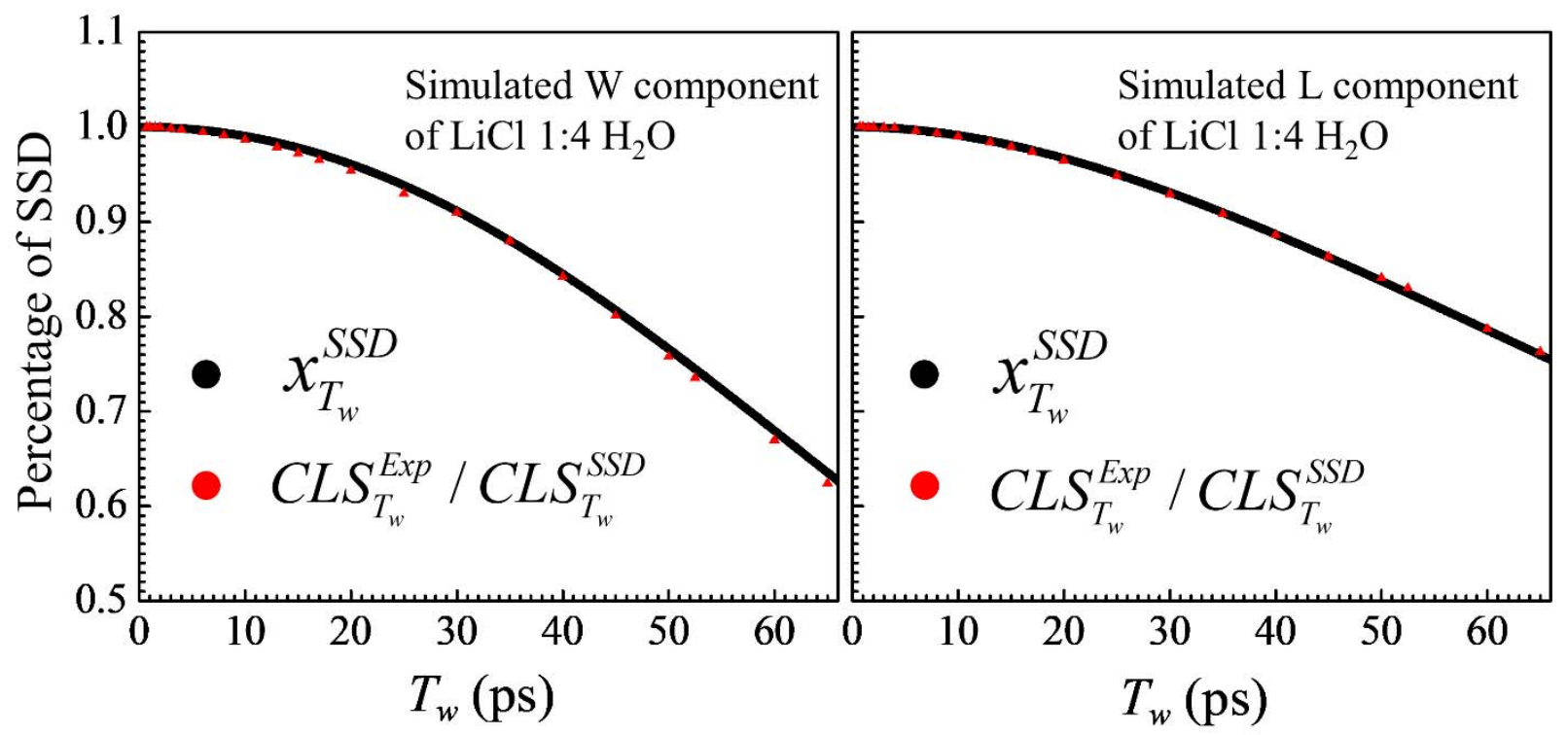

Figure S8. The left and right figures correspond to the simulated W and L components, respectively. Black and red dots, as label suggests, are calculated from exchange model and CLS ratio from 2D spectra, respectively. For both $\mathrm{W}$ and $\mathrm{L}$ components, they are basically on top of each other. 


\section{Reference}

1 Kwak, K.; Zheng, J.; Cang, H.; Fayer, M. D. Ultrafast 2D IR Vibrational Echo Chemical Exchange Experiments and Theory. J. Phys. Chem. B. 2006, 110, 19998-20013.

2 Kwak, K.; Park, S.; Finkelstein, I. J.; Fayer, M. D. Frequency-frequency correlation functions and apodization in two-dimensional infrared vibrational echo spectroscopy: A new approach. $J$. Chem. Phys. 2007, 127, 124503.

3 Kwak, K.; Rosenfeld, D. E.; Fayer, M. D. Taking apart the two-dimensional infrared vibrational echo spectra: More information and elimination of distortions. J. Chem. Phys. 2008, $128,204505$.

4 Tokmakoff, A. Orientational correlation functions and polarization selectivity for nonlinear spectroscopy of isotropic media. 1. Third order. Journal of Chemical Physics 1996, 105, 1-12. 5 Yuan, R.; Yan, C.; Fayer, M. Ion-Molecule Complex Dissociation and Formation Dynamics in LiCl Aqueous Solutions from 2D IR Spectroscopy. J. Phys. Chem. B 2018, 122, 10582-10592. 